\title{
Realizing Discontinuous Wave Functions with Renormalized Short-Range Potentials
}

\author{
Taksu Cheon \\ The Laboratory of Physics, Kochi University of Technology, Tosa Yamada, Kochi 782-8502, Japan \\ email: cheon@mech.kochi-tech.ac.jp, http://www.kochi-tech.ac.jp/ cheond \\ T. Shigehara \\ Department of Information and Computer Sciences, Saitama University, Urawa, Saitama 338-8570, Japan \\ email: sigehara@ics.saitama-u.ac.jp, http://www.me.ics.saitama-u.ac.jp/ ${ }^{\sim}$ sigehara
}

(February 23, 1998)

\begin{abstract}
We show that the most general three-parameter family of point interactions on the line can be expressed as the self-adjoint local operators in terms of three Dirac's delta functions with the renormalized strengths in the disappearing distances. Experimental realization of the Neumann boundary is discussed.
\end{abstract}

KEYWORDS: point interaction, self-adjoint extension, $\delta^{\prime}$ potential, wave function discontinuity, Neumann boundary

PACS Nos: 3.65.-w, 11.10.Gh, 68.65+g

The discontinuity of the gradient of wave functions has been known since the very early days of quantum mechanics. Even today, however, the admissibility of the discontinuity in the wave function itself is not well recognized outside the circle of mathematical physicists. This is mainly due to the esoteric languages employed in formulating the subject, which tends to give an impression that the phenomena are far removed from the experimentally realizable settings.

Let us focus on an example of the simplest, but nontrivial wave function discontinuity that can be found in the free particle quantum mechanics on one-dimensional line with a pointlike interaction, or a point defect [1] 6 . Assuming the time-reversal symmetry, the effect of the interaction is usually expressed in terms of the three parameter connection condition between the wave functions and their derivatives at the left and the right of the location of the defect. Depending on the parameter value, the condition allows the discontinuities both of wave function and its space-derivative. In addition, there can be left-right asymmetry in the connection condition at the defect. In the abstract mathematical treatment, however, no specific prescription is given for the realization of this connection condition as Hamiltonian dynamics. Despite several attempts for the physical interpretation [7.8, the intuitive picture is still lacking. That is evident, for example, in the controversy over its appellation of " $\delta$ ' interaction" (see the discussions in [9, 10]). Also, no experiment can be conceived to check and utilize their effect.

Two recent works 11, 12 on the potential models whose zero-range limit give the full connection condition go some way to address this problem. But the potentials obtained there are non-local and non-Hermitian except in the zero-range limit, thus still leave us puzzled over their relevance to the real-world quantum mechanics. It would be very useful if we can express the point defect in terms of a zero-range limit of well behaving functions.

The objective of this paper is to devise just such potential functions. It is done in terms of zero-distance limit of three or more Dirac's delta functions. This effectively gives the practical prescription to realize the wave function discontinuity as well as its intuitive understanding, since, the delta function in one dimension, in tern, is realizable as a regular limit of small size potential with the volume integral kept constant.

We start by defining a function using three Dirac's delta functions placed on the line separated by small distances $a$;

$$
\xi(x ; v, u, a)=v \delta(x+a)+u \delta(x)+v \delta(x-a) .
$$

The strengths of the delta functions are allowed to be varied as functions of the distance $a$;

$$
v=v(a), \quad u=u(a) \quad \text { as } \quad a \rightarrow 0 .
$$

We look at the quantum-mechanical wave function $\psi(x)$ under the influence of the potential $\xi(x ; v, u, a)$

$$
-\frac{1}{2} \frac{d^{2}}{d x^{2}} \psi(x)+\xi(x ; v, u, a) \psi(x)=E \psi(x) .
$$

The solution of the Schrödinger equation in the intervals $x \in\left[0_{+}, a_{-}\right]$, where $x_{-}$and $x_{+}$respectively signify the location infinitesimally smaller and larger than $x$, can be written in the form

$$
\begin{aligned}
\psi(x) & =\psi(0) \cos k x+\psi^{\prime}\left(0_{+}\right) k^{-1} \sin k x, \\
\psi^{\prime}(x) & =\psi^{\prime}\left(0_{+}\right) \cos k x-\psi(0) k \sin k x,
\end{aligned}
$$

where $k$ is the wave number defined by $k \equiv \sqrt{2 E}$. Assuming that we are interested in the low energy spectra of Eq. (3), we expand Eq. (4) in terms of $k$ to take the leading order, and obtain 


$$
\begin{aligned}
\psi(a) & =\psi(0)+a \psi^{\prime}\left(0_{+}\right) \\
\psi^{\prime}\left(a_{-}\right) & =\psi^{\prime}\left(0_{+}\right)
\end{aligned}
$$

With analogous treatment for $x \in\left[(-a)_{+}, 0_{-}\right]$, we have

$$
\begin{aligned}
\psi(-a) & =\psi(0)-a \psi^{\prime}\left(0_{-}\right), \\
\psi^{\prime}\left((-a)_{+}\right) & =\psi^{\prime}\left(0_{-}\right) .
\end{aligned}
$$

Note that the wave function $\psi(x)$ itself is continuous everywhere at this stage. Combining Eqs. (5) and (6) with the connection conditions at the location of the three Dirac's deltas,

$$
\begin{aligned}
\psi^{\prime}\left((-a)_{-}\right)-\psi^{\prime}\left((-a)_{+}\right) & =-2 v \psi(-a), \\
\psi^{\prime}\left(0_{+}\right)-\psi^{\prime}\left(0_{-}\right) & =2 u \psi(0) \\
\psi^{\prime}\left(a_{+}\right)-\psi^{\prime}\left(a_{-}\right) & =2 v \psi(a)
\end{aligned}
$$

we obtain

$$
\begin{aligned}
\psi^{\prime}\left(a_{+}\right)-\psi^{\prime}\left((-a)_{-}\right) & =B(a)\{\psi(a)+\psi(-a)\} \\
\psi(a)-\psi(-a) & =D(a)\left\{\psi^{\prime}\left(a_{+}\right)+\psi^{\prime}\left((-a)_{-}\right)\right\}
\end{aligned}
$$

where the functions $B(a)$ and $D(a)$ are given by

$$
\begin{aligned}
& B(a)=2 v(a)+\frac{u(a)}{1+a u(a)}, \\
& D(a)=\frac{a}{2 a v(a)+1} .
\end{aligned}
$$

We now take the limit $a \rightarrow 0$ and look at the relations between the quantities

$$
\begin{aligned}
& \psi_{ \pm} \equiv \lim _{a \rightarrow 0} \psi( \pm a), \\
& \psi_{ \pm}^{\prime} \equiv \lim _{a \rightarrow 0} \psi^{\prime}\left(( \pm a)_{ \pm}\right),
\end{aligned}
$$

which express the possible discontinuity of the wave function and its derivative at the location of the interaction which is now a single point, $x=0$.

We can choose the function $v(a)$ and $u(a)$ so that either of $B(a)$ or $D(a)$ becomes zero at $a \rightarrow 0$ limit while keeping the other finite. One obvious choice is to keep $v(a)$ and $u(a)$ constant, that is

$$
v(a)=v_{0}, \quad u(a)=u_{0} .
$$

This results in a potential function

$$
\delta\left(x ; 2 v_{0}+u_{0}\right) \equiv \lim _{a \rightarrow 0} \xi\left(x ; v_{0}, u_{0}, a\right)
$$

which yields the conditions

$$
\begin{aligned}
& \psi_{+}^{\prime}-\psi_{-}^{\prime}=2\left(2 v_{0}+u_{0}\right) \psi_{-}, \\
& \psi_{+}-\psi_{-}=0
\end{aligned}
$$

This means

$$
\delta(x ; v)=v \delta(x)
$$

which is a rather trivial result.

Now comes the second choice, which is obtained by setting $v(a) \rightarrow-1 /(2 a)$ to make the $D(a)$ non-zero and choosing $u(a)$ so that $B(a)$ becomes zero; namely

$$
\begin{aligned}
& v(a)=\frac{1}{2 c}-\frac{1}{2 a} \\
& u(a)=-\frac{1}{a}+\frac{c}{a^{2}} .
\end{aligned}
$$

We then have a potential

$$
\varepsilon(x ; c) \equiv \lim _{a \rightarrow 0} \xi\left(x ; \frac{1}{2 c}-\frac{1}{2 a},-\frac{1}{a}+\frac{c}{a^{2}}, a\right)
$$

which results in

$$
\begin{aligned}
& \psi_{+}^{\prime}-\psi_{-}^{\prime}=0, \\
& \psi_{+}-\psi_{-}=2 c \psi_{-}^{\prime},
\end{aligned}
$$

which is what we wanted; the discontinuity of the wave function.

Several remarks on the discontinuity-inducing potential $\varepsilon(x ; c)$ are in order. In our construction, the function is even with respect to the transposition $x \leftrightarrow-x$. This fact does not seem to favour its interpretation in terms of " $\delta^{\prime}(x)$ " as has been done in the literature. The existence of the third delta in the middle, which is essential to get the convergence, also seems to preclude the interpretation of this function in terms of difference between two deltas. An important special case of $\varepsilon(x ; c)$ is obtained in the limit $c \rightarrow \infty$, which results in $\psi^{\prime}(0)=0$. This is non other than the Neumann boundary condition that separates the system into the two regions $x>0$ and $x<0$. This is contrasted to the Dirichlet boundary condition, which is of course obtained as the $v=\infty$ limit of $\delta(x ; v)$.

The self-adjoint extension theory applied to the quantum mechanics on the line with a point defect gives the most general connection condition for the wave function at the site of defect in the form [6]

$$
\left(\begin{array}{c}
\psi_{+}^{\prime} \\
\psi_{+}
\end{array}\right)=\left(\begin{array}{ll}
-\alpha & -\beta \\
-\delta & -\gamma
\end{array}\right)\left(\begin{array}{l}
\psi_{-}^{\prime} \\
\psi_{-}
\end{array}\right)
$$

with a constraint

$$
\alpha \gamma-\beta \delta=1
$$

The special choice $\alpha=\gamma=-1$ gives the conditions eq. (13) (for $\delta=0$ ) and eq. (17) (for $\beta=0$ ). We therefore have the correspondence

$$
\begin{aligned}
& \left(\begin{array}{cc}
1 & 2 v \\
0 & 1
\end{array}\right) \longleftrightarrow \delta(x ; v), \\
& \left(\begin{array}{cc}
1 & 0 \\
2 c & 1
\end{array}\right) \longleftrightarrow \varepsilon(x ; c) .
\end{aligned}
$$

In order to obtain the potential function which gives the full connection condition, one can work out in a similar fashion as before, with different choice of $v(a)$ and 
$u(a)$, introducing different strengths to the deltas of left and right. But we take different route here, which is easier and, in a sense, gives us better insight into the meaning of the parameter values $\alpha \beta \gamma$ and $\delta$.

The method is based on the matrix identity

$$
\left(\begin{array}{cc}
-\alpha & -\beta \\
-\delta & -\gamma
\end{array}\right)=\left(\begin{array}{cc}
1 & \frac{\alpha+1}{\delta} \\
0 & 1
\end{array}\right)\left(\begin{array}{cc}
1 & 0 \\
-\delta & 1
\end{array}\right)\left(\begin{array}{cc}
1 & \frac{\gamma+1}{\delta} \\
0 & 1
\end{array}\right) \text {. }
$$

Successive application of the matrix to the vector $\left(\psi^{\prime}, \psi\right)$ can be implemented as the point interactions placed next to each other in disappearing distance. With the correspondence eq. (20) in mind, it is easy to convince oneself that the potential function

$$
\begin{aligned}
& \chi(x ; \alpha, \beta, \gamma, \delta) \\
\equiv & \lim _{b \rightarrow 0}\left\{\delta\left(x+b ; \frac{\gamma+1}{2 \delta}\right)+\varepsilon\left(x ; \frac{-\delta}{2}\right)+\delta\left(x-b ; \frac{\alpha+1}{2 \delta}\right)\right\}
\end{aligned}
$$

produces the desired connection condition, eq. (18) at $x=0$. Since no singularity in the coupling is present in the limit $b \rightarrow 0$, one can make $b$ arbitrarily small as long as it doesn't contradict with the definitions eqs.(12) and (16). This means that one can "merge" the two deltas at $x=-b$ and $x=b$ into the two deltas at $x=-a$ and $x=a$ which constitute peripheral flank of $\varepsilon(x ; c)$. One arrives at

$$
\begin{aligned}
& \chi(x ; \alpha, \beta, \gamma, \delta) \\
& =\lim _{a \rightarrow 0}\left\{\delta\left(x+a ; \frac{\gamma-1}{2 \delta}-\frac{1}{2 a}\right)+\delta\left(x ;-\frac{1}{a}-\frac{\delta}{2 a^{2}}\right)\right. \\
& \left.\quad+\delta\left(x-a ; \frac{\alpha-1}{2 \delta}-\frac{1}{2 a}\right)\right\} .
\end{aligned}
$$

It is not difficult to confirm this expression with direct calculation similar to eqs. (7)-(8).

The expressions eqs. 21 - (23) are not valid for $\delta=0$, in which case we resort to the expression

$$
\left(\begin{array}{ll}
-\alpha & -\beta \\
-\delta & -\gamma
\end{array}\right)=\left(\begin{array}{cc}
1 & 0 \\
\frac{\gamma+1}{\beta} & 1
\end{array}\right)\left(\begin{array}{cc}
1 & -\beta \\
0 & 1
\end{array}\right)\left(\begin{array}{cc}
1 & 0 \\
\frac{\alpha+1}{\beta} & 1
\end{array}\right)
$$

which, in terms of the potential function, means

$$
\begin{aligned}
& \chi(x ; \alpha, \beta, \gamma, \delta) \\
= & \lim _{b \rightarrow 0}\left\{\varepsilon\left(x+b ; \frac{\alpha+1}{2 \beta}\right)+\delta\left(x ; \frac{-\beta}{2}\right)+\varepsilon\left(x-b ; \frac{\gamma+1}{2 \beta}\right)\right\} .
\end{aligned}
$$

Combining this and eqs.12) and (16), and again "merging" the neighbouring deltas where appropriate, we obtain an expression which involve five deltas;

$$
\begin{aligned}
& \chi(x ; \alpha, \beta, \gamma, \delta) \\
&=\lim _{a \rightarrow 0}\left\{\delta\left(x+2 a ; \frac{\beta}{\alpha+1}-\frac{1}{2 a}\right)+\delta\left(x+a ;-\frac{1}{a}+\frac{\alpha+1}{2 \beta a^{2}}\right)\right. \\
& \quad+\delta\left(x ; \frac{\beta}{\alpha+1}+\frac{\beta}{\gamma+1}-\frac{\beta}{2}-\frac{1}{a}\right) \\
&\left.+\delta\left(x-a ;-\frac{1}{a}+\frac{\gamma+1}{2 \beta a^{2}}\right)+\delta\left(x-2 a ; \frac{\beta}{\gamma+1}-\frac{1}{2 a}\right)\right\} .
\end{aligned}
$$

The expressions eqs. (24) - (26) are still not applicable to the special case of $\beta=\delta=0$. For this case, we use another expression

$$
\begin{aligned}
\left(\begin{array}{cc}
-\alpha & 0 \\
0 & -\gamma
\end{array}\right) & =\left(\begin{array}{cc}
0 & \rho \\
-\frac{1}{\rho} & 0
\end{array}\right)\left(\begin{array}{cc}
0 & \mp \frac{1}{\rho} \\
\pm \rho & 0
\end{array}\right) \\
& =\left(\begin{array}{cc}
1 & \rho \\
0 & 1
\end{array}\right)\left(\begin{array}{cc}
1 & 0 \\
-\frac{1}{\rho} & 1
\end{array}\right)\left(\begin{array}{ll}
1 & \rho \\
0 & 1
\end{array}\right) \\
& \times\left(\begin{array}{cc}
1 & \mp \frac{1}{\rho} \\
0 & 1
\end{array}\right)\left(\begin{array}{cc}
1 & 0 \\
\pm \rho & 1
\end{array}\right)\left(\begin{array}{cc}
1 & \mp \frac{1}{\rho} \\
0 & 1
\end{array}\right)
\end{aligned}
$$

where $\rho \equiv \sqrt{|\alpha|}=1 / \sqrt{|\gamma|}$ and the composite sign corresponds to the case of $\alpha=\mp|\alpha|$. To obtain the second equality, eq. (21) is applied to the both matrices in the RHS of the first equality. One can implement this expression in potential form as

$$
\begin{aligned}
& \chi(x; \alpha, 0, \gamma, 0) \\
&=\lim _{a \rightarrow 0}\left\{\delta\left(x+2 a ; \pm \frac{1}{2 \rho}-\frac{1}{2 a}\right)+\delta\left(x+a ;-\frac{1}{a} \pm \frac{\rho}{2 a^{2}}\right)\right. \\
& \quad+\delta\left(x ;-\frac{\rho}{2} \pm \frac{1}{2 \rho}-\frac{1}{a}\right) \\
&\left.\quad+\delta\left(x-a ;-\frac{1}{a}-\frac{1}{2 \rho a^{2}}\right)+\delta\left(x-2 a ;-\frac{\rho}{2}-\frac{1}{2 a}\right)\right\} .
\end{aligned}
$$

Thus, the most general connection condition around the point defect in one-dimensional quantum mechanics can be realized as singular, but renormalized zero-distance limit of three (or five) Dirac's delta functions. We note that the above expressions are by no means unique; one can construct expressions with three deltas in place of eqs. (26) and (28) with direct method mentioned before. But more important than the numerical economy is the fact that the expressions eqs. (22), (26) and (28) make the meaning of the asymmetry for $\alpha \neq \gamma$ case very clear; one obtains different results by placing $\delta(x ; v)$ to the left or to the right of $\varepsilon(x ; c)$ even in the zero-distance limit.

We offer a numerical example to illustrate the workings of the realization of the wave function discontinuity. We use finite potential of range $s$ in place of delta as the building block;

$$
\Delta_{s}(x)=\left\{\begin{array}{cl}
\frac{2}{s} \cos ^{2}\left(\frac{\pi}{s} x\right), & x<|s / 2| \\
0, & x>|s / 2|
\end{array}\right.
$$

Then, the potential $E_{a, s}(x ; c)$ defined by

$$
\begin{aligned}
E_{a, s}(x ; c)= & \left(\frac{1}{2 c}-\frac{1}{2 a}\right) \Delta_{s}(x+a) \\
& +\left(-\frac{1}{a}+\frac{c}{a^{2}}\right) \Delta_{s}(x)+\left(\frac{1}{2 c}-\frac{1}{2 a}\right) \Delta_{s}(x-a)
\end{aligned}
$$

becomes a good approximation to, and ultimately converge toward the $\varepsilon(x ; c)$ as one takes the limit $0<s \ll$ $a \rightarrow 0$. 
In Fig. 1, the first four eigenstates of the Schrödinger equation on a line $x \in[-L / 2, L / 2]$ with the potential eq. (30) are shown along with the potential itself. Dirichlet conditions are imposed at the edge, namely, $\psi(L / 2)=$ $\psi(-L / 2)=0$. The value of the coupling is set to be $c=5$. The parameters $a$ and $s$, which are supposed to be smaller than the scale of the problem $L$ (which we arbitrarily set $L=10$ ) are chosen to be $a=0.0333 L$ and $s=0.0012 L$. At this level of "small but finite" $s$ and $a$, one can already observe the discontinuity of the wave function developing around $x=0$ for the second and the fourth states, while the first and the third states show "continuity" because one has $\psi^{\prime}(0)=0$ for these even-parity states. The near degeneracy found between the first and the second states, and also between the third and the fourth states, can be thought of as the sign of the closeness to the Neumann limit $c=\infty$ where the system is divided into the two isolate identical subsystems at $x=0$.
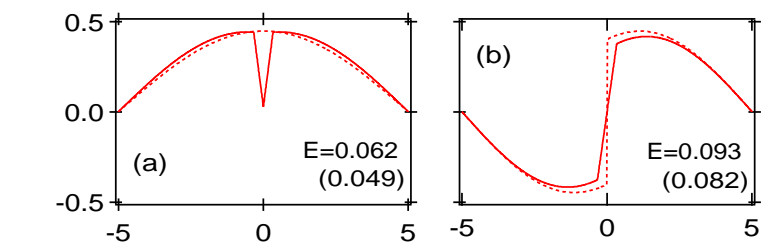

$\supsetneq$
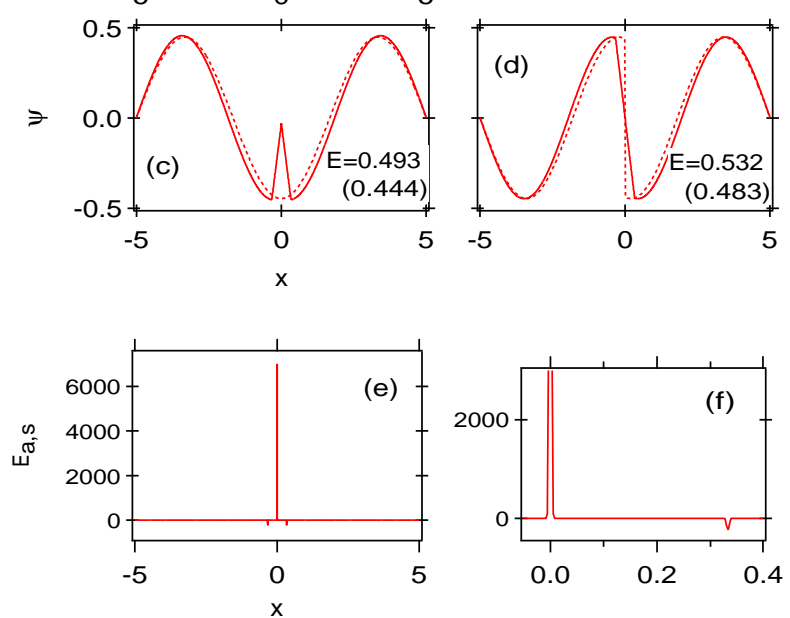

FIG. 1. (a) -(d) show the first four eigenstates of the particle of unit mass on the line $x \in[-L / 2, L / 2]$ with the potential defined by eqs. (26) and (27), which is depicted in (e). The numbers are the energy eigenvalues. Parameters are $c=5, L=10, a=0.0333 L, s=0.0012 L$. The dashed lines show the wave functions (and the numbers in the brackets the eigenvalues) of the predicted $a \rightarrow 0$ limit calculated from the connection condition eq. (14). (e) and (f) depict the potential function in different scales.

This example clearly shows that our procedure to realize the wave function discontinuity is not a mere mathematical abstraction, but something actually realizable in experiments. Recent progress in the quantum device of nanometer scale offers a possible opportunity. We also note that the experiments could be carried out with electro-magnetic and other macroscopic waves, since the results obtained here is applicable to larger classes of linear wave equations other than the Schrödinger equation.

Finally, we place our findings in broader context. Throughout this paper, we have kept our arguments in the language of elementary quantum mechanics. It is not difficult, however, to reformulate the problem in terms of Green's function [13,14. In any case, we believe that our procedures clearly show that there are non-trivial, but experimentally accessible zero-range forces in one dimensional quantum mechanics other than the familiar Dirac's delta function.

In hindsight, it is natural to expect the existence of certain singularity, or the divergence in order to obtain the wave function discontinuity, since the ordinary delta function is known to be "too week" for that purpose. For the theory to be still well-defined with divergent quantity, the renormalization has to be introduced. This is in a sense analogous to the situation in two and three dimensions where one encounters divergence and renormalization in order to define proper point interactions (see, for example, [15] and references therein). We have to add in rush that there is an essential dissimilarity; In two and three dimensions, one can define only one class of zero-range potential which corresponds to $\delta$ function, and there is no analogue to the function $\varepsilon(x ; c)$.

It would be useful to compare the current realization of discontinuity-inducing potential to the earlier approach found in ref [4] which utilizes the separable potential 16] of infinite rank, since the usual $\delta$ function can be thought of as a separable potential of rank one.

The function $\chi(x ; \alpha, \beta, \gamma, \delta)$ belongs to a solvable class of quantum potential while retaining the richness of three parameter dependence. Already, several unusual physical properties are predicted for the systems with discontinuity-inducing potentials [7,8]. Important fact to recall is that all imaginable potentials in one dimension are guaranteed to converge toward $\chi(x ; \alpha, \beta, \gamma, \delta)$ in zerorange limit. As such, it should help us unveil non-trivial aspects of simple quantum systems.

In theoretical treatment of quantum system, the Neumann boundary condition appears as natural as, and at times, more convenient than the Dirichlet boundary. We hope the current work may stimulate the experimental design to materialize and utilize the Neumann boundary condition.

We thank Prof. Izumi Tsutsui for helpful discussions. 
[1] A. Grossmann, H. Høegh-Krohn and M. Mebkhout, J. Math. Phys. 21 (1980) 2376.

[2] F. Gesztesy an W. Kirsch, J. Reine Angew. Math. 362 (1985) 28.

[3] P. Šeba, Czech. J. Phys. B36 (1986) 667.

[4] P. Šeba, Rep. Math. Phys. 24 (1986) 111.

[5] F.G. Gesztesy and H. Holden, J. Phys. A20 (1987) 5157.

[6] S. Albeverio, F. Gesztesy, R. Høegh-Krohn and H. Holden, Solvable Models in Quantum Mechanics, (Springer-Verlag, New York, 1988).

[7] J. E. Avron, P. Exner, Y. Last, Phys. Rev. Lett. 72 (1994) 896.

[8] A. Kiselev, J. Math. Anal. Appl. 212 (1997) 263.

[9] P. Exner, J. Phys. A29 (1996) 87.

[10] F.A.B. Coutinho, Y. Nogami and J.F. Perez, J. Phys. A30 (1997) 3937.

[11] M. Carreau, J. Phys. A26 (1993) 427.

[12] P. R. Chernoff and R. Hughes, J. Funct. Anal. 111 (1993) 92.

[13] C. Grosche, J. Math. Phys. 28 (1995) 99.

[14] D.K. Park, J. Phys. A29 (1996) 6407.

[15] T. Shigehara and T. Cheon, Phys. Rev. E 55 (1997) 6832.

[16] Y. Yamaguchi, Phys. Rev. 95 (1954) 1628. 